\title{
Roles of mitochondrial transcription factor $A$ and microRNA-590-3p in the development of colon cancer
}

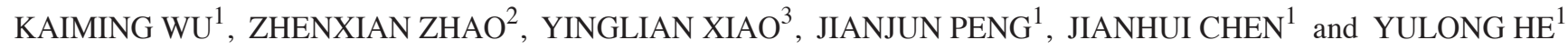 \\ Departments of ${ }^{1}$ Gastrointestinal Surgery, ${ }^{2}$ Pancreato-Biliary Surgery and ${ }^{3}$ Gastroenterology and Hepatology, \\ First Affiliated Hospital of Sun Yat-Sen Univesity, Guangzhou, Guangdong 510080, P.R. China
}

Received September 24, 2015; Accepted October 7, 2016

DOI: $10.3892 / \mathrm{mmr} .2016 .5955$

\begin{abstract}
Mitochondrial transcription factor A (TFAM) participates in the process of mitochondrial DNA replication and transcription. microRNAs (miRNAs) serve an important role in the regulation of gene expression. However, the roles of TFAM and certain miRNAs and their associations in the development of numerous cancer types remain unclear. The current study demonstrated that the expression of TFAM was significantly upregulated in colon cancer compared with the normal tissue, while the expression of miRNA-590-3p (miR-590-3p) was predicted with a high score using miRWalk software, and the luciferase assay demonstrated that TFAM was the direct target of miRNA-590-3p. miR-590-3p exhibited high expression levels in both colon cancer tissue and the SW480 cell line. Furthermore, downregulated expression of miR-590-3p significantly inhibited the growth of SW480 cells, which was consistent with results indicating downregulated expression of TFAM in SW480 cells from a previous study. In summary, the results of the current study concluded that miR-590-3p, via direct targeting of TFAM, may serve an important role in the tumorigenesis of colon cancer, and may be a promising target for colon cancer therapeutics.
\end{abstract}

\section{Introduction}

The mortality rate associated with colon cancer ranks as the fourth highest of different cancer types worldwide (1). According to the data from Globocan, an international cancer research organization governed by the World Health Organization, there were 694,000 cases of colon cancer-associated mortality during 2012, and 52\% of these were from developing countries. Eastern Europe had the highest mortality rate at

Correspondence to: Professor Yulong He, Department of Gastrointestinal Surgery, First Affiliated Hospital of Sun Yat-Sen Univesity, 58 Zhong Shan Er Road, Guangzhou, Guangdong 510080, P.R. China

E-mail: ylh@medmail.com.cn

Key words: mitochondrial transcription factor A, miRNA-590-3p, SW480 cells, colon cancer
$20.3 \%$ in males and $11.7 \%$ in females, while the Western Africa had the lowest mortality rate at $3.5 \%$ in males and $3.0 \%$ in females. There are 253,000 recently diagnosed cases in China, predominantly in 50-55 year olds. The morbidity is increasing in males, and is marginally reducing in females in China (2).

TFAM, which is a member of the high mobility group (HMG) box protein family, participates in the process of mitochondrial DNA (mtDNA) replication and transcription (3-5). In numerous types of cancer cells, HMG proteins are upregulated (6). In addition, mitochondria are critical for cancer cell involvement in the regulation of cancer cell survival and growth. The Warburg effect is essential for the survival and proliferation of cancer cells by mitochondrial uncoupling regulating the metabolic shift to aerobic glycolysis (7). It has been reported that the high expression of TFAM is associated with a poor prognosis in multiple malignant tumors, including ovarian cancer, endometrial carcinoma and colon cancer $(8,9)$. It has been reported that the TFAM protein multimerizes and binds to mtDNA which regulates the expression of nuclear genes, suggesting that the TFAM levels may be increased in cancer cells and be associated with malignant progression and proliferative activity (10-13). However, the roles of TFAM remain to be fully elucidated in colon cancer cells.

miRNAs, which are small, endogenous and non-coding RNAs, can interact with target sites in the 3'-untranslated regions (UTRs) of mRNA and then inhibit the expression of targeted genes $(14,15)$. Those targeted genes are involved in the regulation of multiple biological processes, including the pathogenesis of a variety of types of human cancer (16-22). miR-590-3p has been reported to be involved in mediating the expression of autoimmune genes and neuronal death $(23,24)$. However, whether or not miR-590-3p is associated with colon cancer remains unclear. Thus, the present study aimed to elucidate the roles of TFAM and miR-590-3p and their association in colon cancer cells.

\section{Materials and methods}

Human tissue specimens. The present study was approved by the ethics committee of the First Affiliated Hospital of Sun Yat-Sen University (Guangzhou, China). There were 30 cases of colectomy for colon cancer performed by the First Affiliated Hospital of Sun Yat-Sen University between March and 
April 2013. Samples were collected from the specimens at the mucosa that were $10 \mathrm{~cm}$ adjacent to the colon cancer (the control group), and from the cancer tissue itself (the experiment group). Patients provided written informed consent.

Patient selection criteria: i) Primary colon cancer diagnosed by histopathology; ii) underwent colon cancer resection and lymph gland cleaning; iii) a minimum of 12 lymph glands undergoing biopsy subsequent to surgery; iv) detailed pathological follow-up records.

Patient rejection criteria: i) History of an additional primary cancer; ii) distant metastasis (peritoneum, liver, brain, etc.); iii) underwent neoadjuvant chemotherapy and other etiotropic therapy; iv) succumbed to disease.

A total of 30 patients were selected, with an average age of 59 years old and including 18 males (60\%) and 12 females (40\%). The cancer locations were identified, with 15 cases $(50 \%)$ at the sigmoid colon, 7 cases (23.3\%) at the descending colon and 8 cases $(26.7 \%)$ at the ascending colon. According to the TNM classification in the 3rd edition of NCCN 2014 (25), there were 28 phase III patients $(93.3 \%)$ and 2 phase II patients $(6.7 \%)$.

Specimen collection involved obtaining $\sim 5 \times 5 \mathrm{~mm}$ samples from the cancer tissues and from mucosa that was $10 \mathrm{~cm}$ adjacent to the cancer (normal colon tissue) within $20 \mathrm{~min}$ of removal from the body. The specimens were placed into two tubes and stored in liquid nitrogen; one to be used for protein extraction and the other for RNA extraction.

Cell culture. Normal human colon cells were purchased from the American Type Culture Collection (CCD-18Co; ATCC ${ }^{\circledR}$ CRL-1459 ${ }^{\mathrm{Tm}}$; Manassas, VA, USA). SW480 cells (Institute of Cell Biology, Chinese Academy of Sciences, Shanghai, China) was cultured in RPMI-1640 medium with $10 \%$ fetal bovine serum (FBS) and $1 \%$ penicillin/streptomycin (Xinxianfeng Pharmaceutical Industry Limited Company, Shanghai, China) at $37^{\circ} \mathrm{C}$, with $5 \% \mathrm{CO}_{2}$. The lentivirus, lentiviral vector and Dual-Luciferase Reporter Assays were purchased from GeneCopoeia, Inc. (Guangzhou, China).

miRNA prediction. Subsequent to inputting TFAM to miRWalk 2.0 (http://zmf.umm.uni-heidelberg. de/apps/zmf/mirwalk2/) as target gene, matching miRNA results were indicated by high scores in Microt4, miRanda, miRBridge, miRDB, miRMap, miRNAMap, PicTar2, PITA, RNA22, RNAhybrid and TargetScan. The target miRNAs were verified in TargetScan and miRanda.

$R N A$ extraction and reverse transcription-quantitative polymerase chain reaction $(R T-q P C R)$. RNA was extracted from the normal colon tissues (30 specimens), colon cancer tissues (30 specimens), normal colon cells and SW480 cells using TRIzol reagent (Invitrogen; Thermo Fisher Scientific, Inc., Waltham, MA, USA), according to the manufacturer's instructions. The RNA integrity was then assessed. A TaqMan RT-qPCR miRNA assay (Applied Biosystems; Thermo Fisher Scientific, Inc.) was performed to detect the expression levels of mature miR-590-3p. The relative expression of mature miR-590-3p levels normalized to U6 endogenous control was determined using the $2^{-\Delta \Delta \mathrm{Cq}}$ method (26). Each measurement was performed in triplicate. In order to detect the target genes (mRNA expression), $1 \mu \mathrm{g}$ total RNA was reverse transcribed to cDNA using SuperScript ${ }^{\mathrm{TM}}$ III
First-Strand Synthesis Super Mix (Invitrogen; Thermo Fisher Scientific, Inc.). SYBR green qPCR was performed using the Bio-Rad iQ5 PCR detection system (Bio-Rad Laboratories, Inc., Hercules, CA, USA) with the following gene-specific primers: miR-590-3p, forward 5'-AAAGATTCCAAGAAGCTAAGG GTG-3', reverse 5'-CCTAACTGGTTTCCTGTGCCTA-3'; and U6, forward 5'-TGCGGGTGCTCCGCTTCGGCAGC-3' and reverse 5'-CAGTGCAGGGTCCGAGGT-3'. PCR cycling conditions were as follows: Initial cycle at $95^{\circ} \mathrm{C}$ for $3 \mathrm{~min}$, followed by pre-denaturation at $95^{\circ} \mathrm{C}$ for $15 \mathrm{sec}$, denaturation at $95^{\circ} \mathrm{C}$ for $5 \mathrm{sec}$ and extension at $60^{\circ} \mathrm{C}$ for $30 \mathrm{sec}$, for $45 \mathrm{cycles}$, and a final extension step at $70^{\circ} \mathrm{C}$ for $30 \mathrm{~min}$.

Correlation analysis. Correlation analysis was conducted for the expression of miR-590-3p mRNA (normalized to U6) and the expression of TFAM mRNA (normalized to $\beta$-actin) in the same specimen.

Dual luciferase reporter assays. To generate the reporter vectors bearing miRNA-binding sites, a normal and mutated 3'-UTR of TFAM was sub-cloned using PCR-based methods (27). The constructs were inserted into the multiple cloning sites downstream of the luciferase gene in the psiCHECK-2 luciferase miRNA expression reporter vector. For the luciferase assay, $1 \times 10^{5}$ cells were cultured to $70-80 \%$ confluence in 24 -well plates and co-transfected with psiCHECK-2-TFAM-3'-UTR or psiCHECK2-mut-TFAM-3'-UTR vector plus $50 \mathrm{nM}$ miR-590-3p or $100 \mathrm{mM}$ miR-590-3p inhibitor using Lipofectamine 2000 (Invitrogen; Thermo Fisher Scientific, Inc.), according to the manufacturer's instructions. The cells were incubated with transfection reagent/DNA complex for $5 \mathrm{~h}$ at $37^{\circ} \mathrm{C}$ and refreshed with fresh medium containing $10 \% \mathrm{FBS}$. At $48 \mathrm{~h}$ post-transfection, firefly and Renilla luciferase activities were evaluated using the Dual-Luciferase Reporter Assay system and the Renilla luciferase activity was normalized to firefly luciferase activity.

Western blotting. The expression of TFAM in SW480 cells was determined subsequent to transfection by miR-590-3p lentivirus or anti-miR-590-3p lentivirus by western blotting. Following $48 \mathrm{~h}$-transfection and $24 \mathrm{~h}$-cell culture, the cells were solubilized in cold RIPA lysis buffer and then separated with $10 \%$ SDS-PAGE. Following this, the proteins were transferred onto PVDF membranes. The membranes were blocked in 5\% skimmed dried milk in phosphate-buffered saline and then incubated overnight with primary antibodies for TFAM (1:500; cat. no. PB9447; Boster Biological Technology, Pleasanton, CA, China), and $\beta$-actin (1:500; cat. no. PA1872; Boster Biological Technology). Subsequent to incubation with goat polyclonal anti-rabbit secondary antibodies (1:4,000; cat. no. A-11034; Invitrogen; Thermo Fisher Scientific, Inc.), the immune complexes were detected using the enhanced chemiluminescence method. The blots were visualized by autoradiography using preflashed Kodak XAR film (Kodak Japan Ltd., Tokyo, Japan). The results were analyzed with Gel-Pro Analyzer, version 4.0 (Silk Scientific, Inc., Orem, UT USA) subsequent to visualization.

MTTassays.Subsequent to transfection, the miR-SCR-SW480, miR-590-3p-SW480 and anti-miR-590-3p-SW480 cell groups were cultured at a density of 5,000 cells/well. A total 
of $10 \mu \mathrm{l}$ MTT (5 mg/ml; Promega Corporation) was added to the medium at $0,24,48,72,96$ and $120 \mathrm{~h}$. The cells were then incubated for $4 \mathrm{~h}$ at $37^{\circ} \mathrm{C}$. The medium was removed and $100 \mu \mathrm{l}$ dimethyl sulfoxide was added into each well. The plate was gently rotated on an orbital shaker for $15 \mathrm{~min}$ to completely dissolve the precipitation. The absorbance was detected at $490 \mathrm{~nm}$ with a microplate reader (Bio-Rad Laboratories, Inc.).

Statistical analysis. All experiments were performed three times in triplicate. The data were analyzed with Student's two-tailed t-test. All statistical analyses were performed using SPSS software, version 17.0 (SPSS, Inc., Chicago, IL, USA). $\mathrm{P}<0.05$ was considered to indicate a statistically significant difference.

\section{Results}

mRNA expression of TFAM is increased in colon cancer tissues vs. adjacent normal colon tissues. mRNA expression of TFAM in tumor tissue samples and adjacent normal tissues was examined using the RT-qPCR assay. The average expression levels of TFAM were significantly increased in colon cancer tissues compared with adjacent normal colon tissues (Fig. 1; $\mathrm{P}<0.0001$ ).

Correlation analysis between TFAM and miR-590-3p. miR-590-3p exhibited a high prediction score (the highest score is 9) in miRWalk 2.0 (Fig. 2A), identifying TFAM as the target gene. The 3'-UTR of miR-590-3p was correlated with TFAM in miRanda (Fig. 2B) and TargetScan (Fig. 2C). The results of the luciferase assay for miR-590-3p are presented in Fig. 2D and the data indicated that the Renilla/firefly value of luciferase was significantly lower in the miR-590-3p treatment cells following transfection with the 3'-UTR of the TFAM gene (Psi-wtTFAM; $\mathrm{P}=0.005$ ), while the Renilla/firefly value of luciferase exhibited no differences following transfection with the mutated 3'-UTR of TFAM (Psi-mutTFAM) compared with the control (Fig. 2D; $\mathrm{P}=0.7870$ ). These data suggested the 3 '-UTR of TFAM is the direct target of miR-590-3p. The expression of miR-590-3p (normalized to U6) in normal colon and colon cancer tissues exhibited positive correlation with that of TFAM (normalized to $\beta$-actin) in the same specimens, correlation coefficent $r=0.9456$, $\mathrm{P}<0.0001$ (Fig. 2E).

mRNA expression of miR-590-3p is increased in colon cancer tissues and cells. mRNA expression of miR-590-3p in tumor tissue samples and adjacent normal tissues was examined through the RT-qPCR assay. The expression of miR-590-3p in the colon cancer tissues was significantly increased compared with adjacent normal colon tissues (Fig. 3A; $\mathrm{P}<0.05$ ). In addition, the mRNA expression of miR-590-3p in SW480 cells was significantly increased compared with the normal colon cells (Fig. 3A; P<0.05).

The miR-590-3p inhibitor effectively suppressed miR-590-3p expression. Based on the fact that the expression level of miR-590-3p is upregulated in colon cancer tissues and cells, the miR-590-3p inhibitor was transfected into SW480 cells. Subequently, cells were harvested for RT-qPCR. The results indicated that the mRNA expression of miR-590-3p was

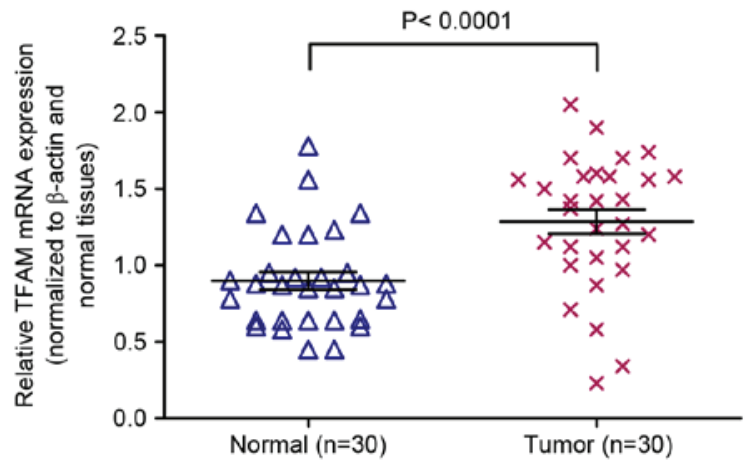

Figure 1. mRNA expression of TFAM in tumor tissue samples and adjacent normal tissues was examined through reverse transcription-quantitative polymerase chain reaction assay. The average expression levels of TFAM were significantly increased in colon cancer tissues compared with adjacent normal colon tissues $(\mathrm{P}<0.0001)$. TFAM, mitochondrial transcription factor A.

increased in SW480 cells transfected with miR-590-3p lentivirus compared with the control (Fig. 4A; $\mathrm{P}<0.0001$ ). The expression of miR-590-3p mRNA was reduced in the SW480 cells transfected with the anti-miR-590-3p lentivirus compared with that of the control (Fig. 4B; $\mathrm{P}=0.0008$ ).

Inhibition of miR-590-3p suppressed TFAM protein expression. To demonstrate the role of $\mathrm{miR}-590-3 \mathrm{p}$ in regulating TFAM protein expression in SW480 cells. Western blotting was conducted subsequent to transfection with miR-590-3p inhibitor (anti-miR-590-3p) or miR-SCR. The expression of TFAM was significantly increased in the SW480 cells transfected with miR-590-3p lentivirus when compared with that of the control (Fig. 5A and B; $\mathrm{P}<0.0001$ ). The expression of TFAM was observed to be significantly reduced in the SW480 cells transfected with the anti-miR-590-3p lentivirus compared with that of the control (Fig. 5C and D; $\mathrm{P}=0.0002$ ).

Inhibition of miR-590-3p suppressed the proliferation of SW480 cells. In order to demonstrate the role of miR-590-3p in the regulation of SW480 cell proliferation, MTT assays were conducted subsequent to transfection with the miR-590-3p inhibitor (anti-miR-590-3p) or miR-SCR. In the MTT assays, the proliferation of the four groups were observed to have no significant differences in the first $96 \mathrm{~h}$ following transfection. However, a growth inhibition of SW480 cells transfected with anti-miR-590-3p compared with controls $(\mathrm{P}<0.0001)$ was observed $120 \mathrm{~h}$ after transfection. The proliferation of SW480 cells with miR-590-3p transfection was greater than that of the controls $(\mathrm{P}<0.0001)$ while that between SW480 and miR-SCR-SW480 exhibited no differences $(\mathrm{P}>0.05) 120 \mathrm{~h}$ subsequent to transfection (Fig. 6).

\section{Discussion}

It has been reported that miRNAs serve significant roles in tumorigenesis. Studies have demonstrated that certain miRNAs are significantly deregulated in gastric cancer, bladder cancer, endometrial carcinoma and may function as tumor regulators (28-30). miRNAs represent a class of small interfering RNAs that can silence genes. miRNAs hybridize to partially 
A

miRwalk 2.0

Gene EntrezID RefseqID miRNA miRwalk Microt4 miRanda mirbridge miRDB miRmap miRNAmap Pictar2 PITA RNA22 RNAhybrid Targetscan SUM

IFAM $7019 \quad$ NM_003201 $\frac{\frac{\text { hsa. }}{\text { miR. }}}{\underline{590-3 p}}$

$\mathbf{B}$

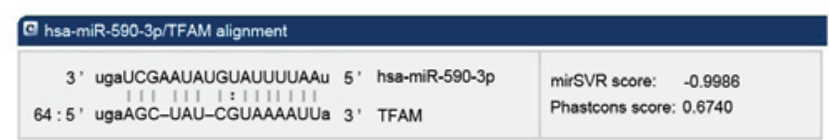

C

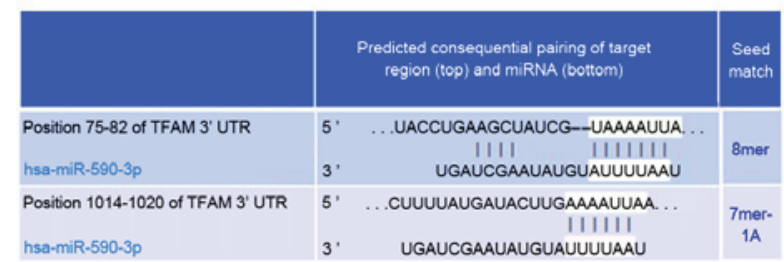

$\mathbf{E}$

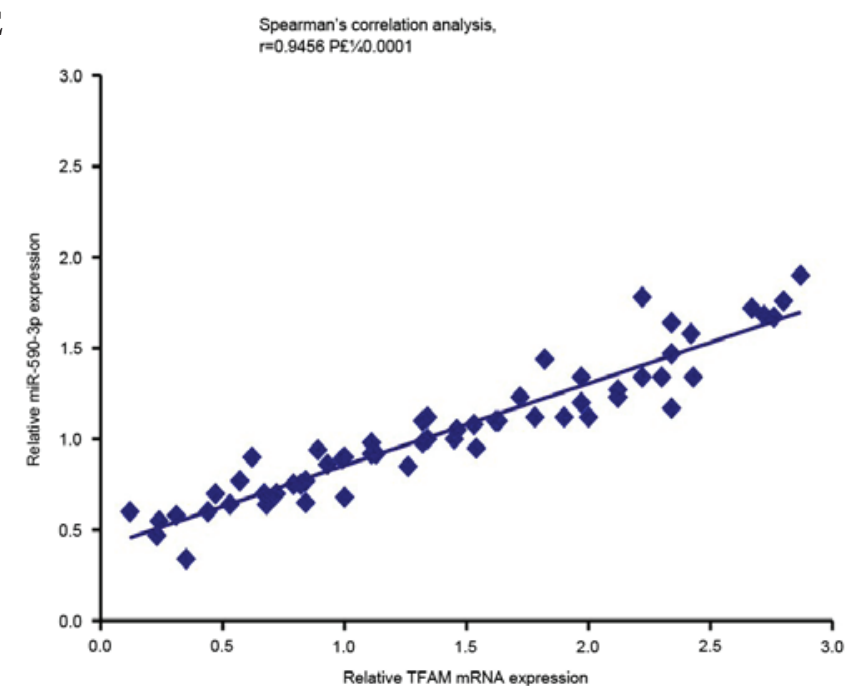

Figure 2. Correlation analysis between TFAM and miR-590-3p. (A) miR-590-3p demonstrated a high prediction score (the highest score is 9) in miRWalk 2.0, matching TFAM as the target gene. The 3'-UTR of miR-590-3p matched with TFAM in (B) miRanda and (C) TargetScan. (D) Luciferase assay results for miR-590-3p; the data indicated that the renilla/firefly value of luciferase was significantly lower in miR-590-3p-treatment cells following transfection with the 3'-UTR of the TFAM gene (Psi-wtTFAM, P=0.005), while the renilla/firefly value of luciferase exhibited no difference following transfection with the mutated 3'-UTR of TFAM (Psi-mutTFAM) compared with the control ( $\mathrm{P}=0.7870$ ). These data suggested the 3'-UTR of TFAM is a direct target of miR-590-3p. (E) The expression of miR-590-3p (normalized to U6) in normal colon and colon cancer tissues were observed to exhibit a positive correlation with that of TFAM (normalized to $\beta$-actin) in the same specimen, correlation coefficent $\mathrm{r}=0.9456, \mathrm{P}<0.0001$. TFAM, mitochondrial transcription factor A; miR, microRNA; UTR, untranslated region; control, SW480 cells; miR-SCR, SW480 cells transfected with miR-SCR lentivirus; miR-590-3p, SW480 cells transfected with miR-590-3p lentivirus.
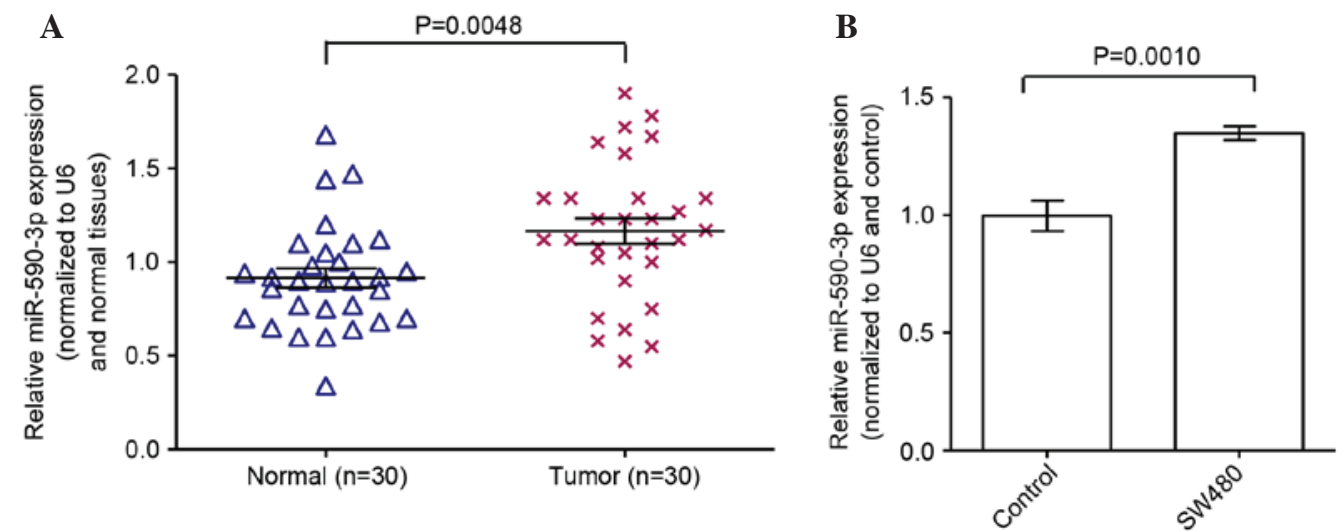

Figure 3. mRNA expression of miR-590-3p in tumor tissue samples and adjacent normal tissues examined through the reverse transcription-quantitative polymerase chain reaction assay. (A) The expression of miR-590-3p in the colon cancer tissues was significantly higher compared with adjacent normal colon tissues $(\mathrm{P}<0.05)$. (B) In addition, the mRNA expression of miR-590-3p in SW480 cells was significantly higher compared with the normal colon cells $(\mathrm{P}<0.05)$. miR, microRNA. 
A

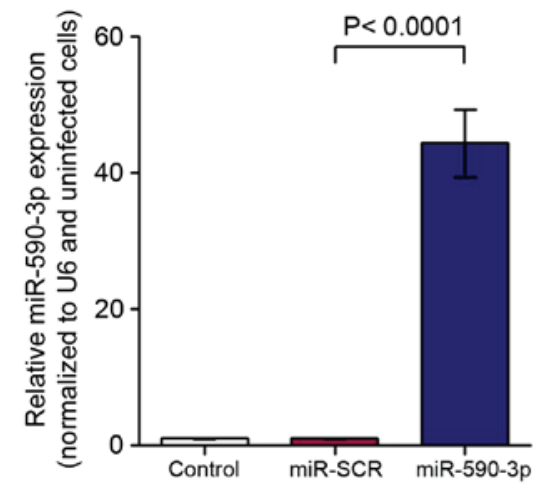

B

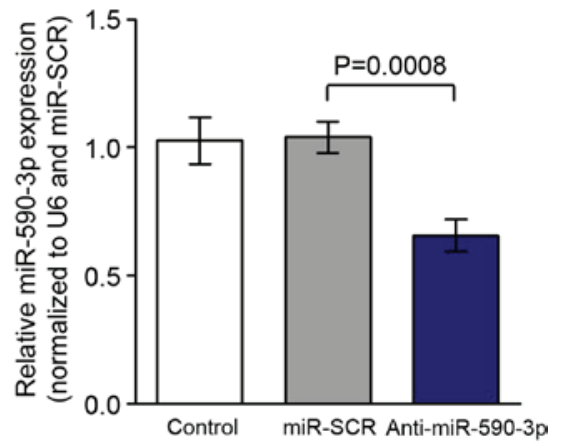

Figure 4. The results of reverse transcription-quantitative polymerase chain reaction demonstrated that (A) mRNA expression of miR-590-3p was higher in SW480 cells transfected with miR-590-3p lentivirus vs. the control $(\mathrm{P}<0.0001)$. (B) The expression of miR-590-3p mRNA was reduced in SW480 cells transfected with anti-miR-590-3p lentivirus vs. the control, $(\mathrm{P}=0.0008)$. miR, microRNA; control, SW480 cells; miR-SCR, SW480 cells transfected with miR-SCR lentivirus; miR-590-3p, SW480 cells transfected with miR-590-3p lentivirus; anti-miR-590-3p, SW480 cells transfected with the anti-miR-590-3p lentivirus.

A

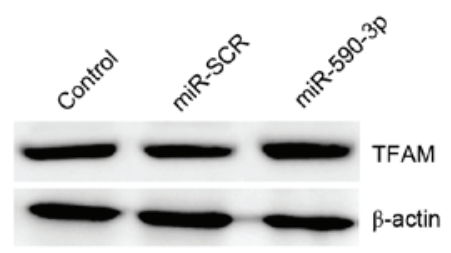

C

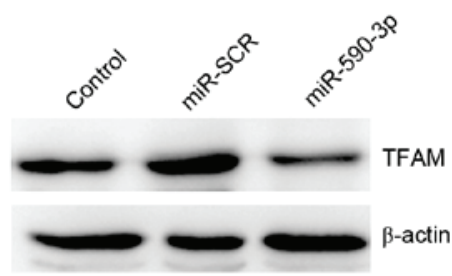

B

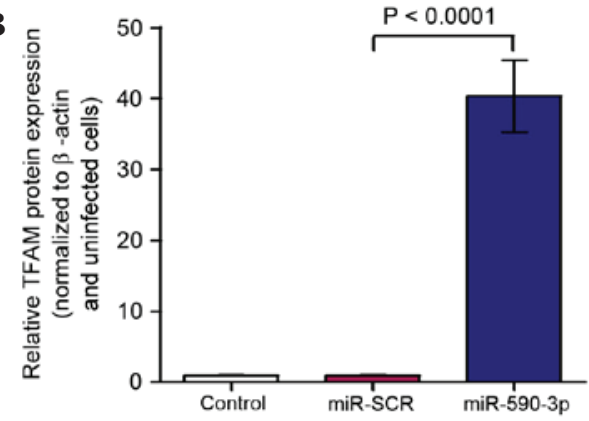

D

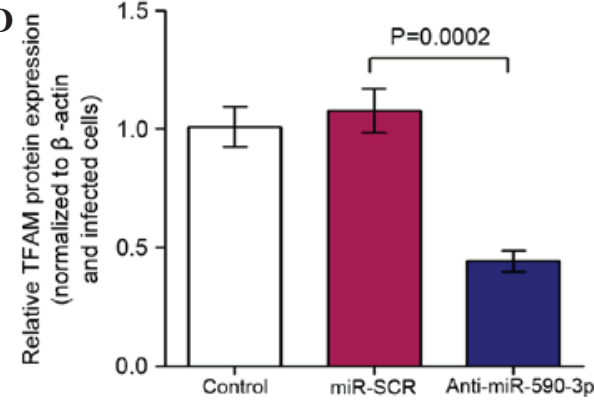

Figure 5. Western blotting was conducted following transfection with the miR-590-3p inhibitor (anti-miR-590-3p) or miR-SCR. (A and B) TFAM expression was significantly higher $(\mathrm{P}<0.0001)$ in SW480 cells transfected with miR-590-3p lentivirus vs. the control (A, western blots; B, quantification). (C and D) The expression of TFAM was significantly ( $\mathrm{P}=0.0002)$ lower in SW480 cells transfected with anti-miR-590-3p lentivirus when compared with the control (C, western blots; D, quantification). miR, microRNA; TFAM, mitochondrial transcription factor A; control, SW480 cells; miR-SCR, SW480 cells transfected with miR-SCR lentivirus; miR-590-3p, SW480 cells transfected with miR-590-3p lentivirus; anti-miR-590-3p, SW480 cells transfected with the anti-miR-590-3p lentivirus.

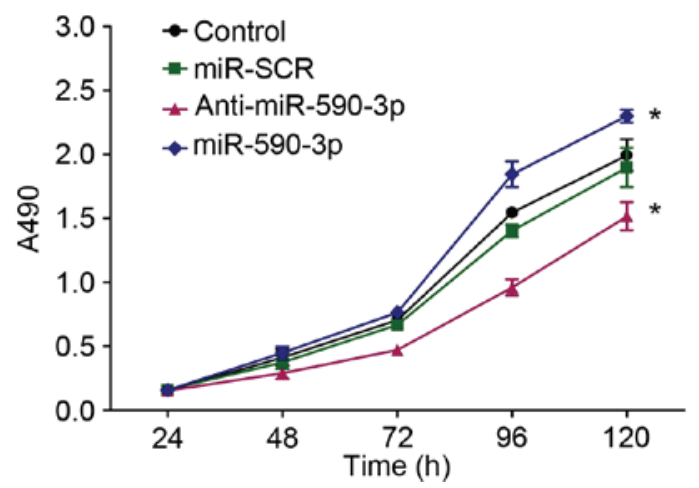

Figure 6. MTT assays were conducted after transfection with miR-590-3p inhibitor (anti-miR-590-3p) or miR-SCR. No significant differences in proliferation were observed within the first $96 \mathrm{~h}$ after transfection. Growth inhibition of SW480 cells with anti-miR-590-3p transfection vs. controls ("P<0.0001) was observed $120 \mathrm{~h}$ after transfection. The proliferation of SW480 with miR-590-3p transfection was higher than controls ( $\mathrm{P}<0.0001$ ) while that between SW480 and miR-SCR-SW480 exhibited no significant differences ( $\mathrm{P}>0.05) 120 \mathrm{~h}$ after transfection. miR, microRNA; control, SW480 cells; miR-SCR, SW480 cells transfected with miR-SCR lentivirus; miR-590-3p, SW480 cells transfected with miR-590-3p lentivirus; anti-miR-590-3p, SW480 cells transfected with the anti-miR-590-3p lentivirus. 
complementary binding sites that are typically located in the 3-UTR of target mRNAs and regulate their expression (15).

TFAM can promote mtDNA transcription using mitochondrial RNA polymerase in a promoter-specific manner. The replication of mammalian mtDNA is hypothesized to be coupled with transcription, and TFAM has been suggested to be essential for the replication of mtDNA (10-12). TFAM exhibited high expression levels in colon cancer and upregulated the proliferation of SW480 cells in a previous study (31). Although the mechanism of TFAM overexpression remains unclear, one possible mechanism maybe via the regulation of miRNAs. Therefore, TFAM was selected as the target gene in order to identify the regulatory miRNA. MiRWalk 2.0 is a comprehensive database providing information on miRNAs from humans, mice and rats, detailing their predicted and validated binding sites on their target genes. MiRWalk summarized the predition frequencies in Microt4, miRanda, miRBridge, miRDB, miRMap, miRNAMap, PicTar2, PITA, RNA22, RNAhybrid and TargetScan and the output was a score. Subsequently, miR-590-3p was selected for further study due to its high prediction score. As predicted, the present study indicated that TFAM was the direct target of miR-590-3p in luciferase reporter assays.

In addition, high expression of miRNA-150-3p was identified in colon cancer tissue, which was positively correlated with TFAM. It was indicated that miRNA-150-3p may enhance the expression of TFAM. The overexpression of miRNA-150-3p may promote the aberrant expression of TFAM, thus contributing to the progression of colon cancer. In addition, the suppressive effect against proliferation was confirmed by MTT assays, which investigated the downregulation of miR-590-3p.

In summary, the present study identified the interaction of miR-590-3p and TFAM in colon cancer. TFAM was identified as a target of miR-590-3p, and miR-590-3p enhanced the proliferation of SW480 cells. miR-590-3p was suggested as a novel target for knockdown.

\section{References}

1. http://www.who.int/mediacentre/factsheets/fs297/en/. Accessed on 29 August 2012.

2. Bray F, Ren JS, Masuyer E and Ferlay J: Global estimates of cancer prevalence for 27 sites in the adult population in 2008 . Int J Cancer 132: 1133-1145, 2013.

3. Bonawitz ND, Clayton DA and Shadel GS: Initiation and beyond: Multiple functions of the human mitochondrial transcription machinery. Mol Cell 24: 813-825, 2006.

4. Parisi MA and Clayton DA: Similarity of human mitochondrial transcription factor 1 to high mobility group proteins. Science 252 : 965-969, 1991.

5. Litonin D, Sologub M, Shi Y, Savkina M, Anikin M, Falkenberg M, Gustafsson CM and Temiakov D: Human mitochondrial transcription revisited: Only TFAM and TFB2M are required for transcription of the mitochondrial genes in vitro. J Biol Chem 285: 18129-18133, 2010.

6. Tang D, Kang R, Zeh HJ III and Lotze MT: High-mobility group box 1 and cancer. Biochim Biophys Acta 1799: 131-140, 2010.

7. Baffy G: Uncoupling protein-2 and cancer. Mitochondrion 10: 243-252, 2010.

8. Yoshida Y, Hasegawa J, Nezu R, Kim YK, Hirota M, Kawano K, Izumi $\mathrm{H}$ and Kohno K: Clinical usefulness of mitochondrial transcription factor a expression as a predictive marker in colorectal cancer patients treated with FOLFOX. Cancer Sci 102: 578-582, 2011

9. Toki N, Kagami S, Kurita T, Kawagoe T, Matsuura Y, Hachisuga T, Matsuyama A, Hashimoto H, Izumi $\mathrm{H}$ and Kohno K: Expression of mitochondrial transcription factor A in endometrial carcinomas: Clinicopathologic correlations and prognostic significance. Virchows Arch 456: 387-393, 2010.
10. Alam TI, Kanki T, Muta T, Ukaji K, Abe Y, Nakayama H, Takio K, Hamasaki N and Kang D: Human mitochondrial DNA is packaged with TFAM. Nucleic Acids Res 31: 1640-1645, 2003.

11. Kaufman BA, Durisic N, Mativetsky JM, Costantino S, Hancock MA, Grutter P and Shoubridge E: The mitochondrial transcription factor TFAM coordinates the assembly of multiple DNA molecules into nucleoid-like structures. Mol Biol Cell 18: 3225-3236, 2007.

12. Xu S, Zhong M, Zhang L, Wang Y, Zhou Z, Hao Y, Zhang W, Yang X, Wei A, Pei L and Yu Z: Overexpression of Tfam protects mitochondria against beta-amyloid-induced oxidative damage in SH-SY5Y cells. FEBS J 276: 3800-3809, 2009.

13. Aydin J, Andersson DC, Hänninen SL, Wredenberg A, Tavi P, Park CB, Larsson NG, Bruton JD and Westerblad H: Increased mitochondrial $\mathrm{Ca} 2+$ and decreased sarcoplasmic reticulum $\mathrm{Ca} 2+$ in mitochondrial myopathy. Hum Mol Genet 18: 278-288, 2009.

14. Kidani A, Izumi H, Yoshida Y, Kashiwagi E, Ohmori H, Tanaka T, Kuwano $\mathrm{M}$ and Kohno K: Thioredoxin2 enhances the damaged DNA binding activity of mtTFA through direct interaction. Int J Oncol 35: 1435-1440, 2009.

15. Bartel DP: MicroRNAs: Target recognition and regulatory functions. Cell 136: 215-233, 2009.

16. Petersen CP, Bordeleau ME, Pelletier J and Sharp PA: Short RNAs repress translation after initiation in mammalian cells. Mol Cell 21: 533-542, 2006.

17. Adam L, Zhong M, Choi W, Qi W, Nicoloso M, Arora A, Calin G, Wang H, Siefker-Radtke A, McConkey D, et al: MiR-200 expression regulates epithelial-to-mesenchymal transition in bladder cancer cells and reverses resistance to epidermal growth factor receptor therapy. Clin Cancer Res 15: 5060-5072, 2009.

18. Saito Y, Suzuki H, Tsugawa H, Nakagawa I, Matsuzaki J, Kanai Y and Hibi T: Chromatin remodeling at Alu repeats by epigenetic treatment activates silenced microRNA-512-5p with down regulation of Mcl-1 in human gastric cancer cells. Oncogene 28: 2738-2744, 2009.

19. Mazar J, DeYoung K, Khaitan D, Meister E, Almodovar A, Goydos J, Ray A and Perera RJ: The regulation of miRNA-211 expression and its role in melanoma cell invasiveness. PLoS One 5: e13779, 2010.

20. Di Leva G, Piovan C, Gasparini P, Ngankeu A, Taccioli C, Briskin D, Cheung DG, Bolon B, Anderlucci L, Alder H, et al: Estrogen mediated-activation of miR-191/425 cluster modulates tumorigenicity of breast cancer cells depending on estrogen receptor status. PLoS Genet 9: e1003311, 2013.

21. Rivas MA, Venturutti L, Huang YW, Schillaci R, Huang TH and Elizalde PV: Downregulation of the tumor-suppressor miR-16 via progestin-mediated oncogenic signaling contributes to breast cancer development. Breast Cancer Res 14: R77, 2012.

22. Kalinowski FC, Giles KM, Candy PA, Ali A, Ganda C, Epis MR, Webster RJ and Leedman PJ: Regulation of epidermal growth factor receptor signaling and erlotinib sensitivity in head and neck cancer cells by miR-7. PLoS One 7: e47067, 2012.

23. Villa C, Fenoglio C, De Riz M, Clerici F, Marcone A, Benussi L, Ghidoni R, Gallone S, Cortini F, Serpente M, et al: Role of hnRNP-A1 and miR-590-3p in neuronal death: Genetics and expression analysis in patients with Alzheimer disease and frontotemporal lobar degeneration. Rejuvenation Res 14: 275-281, 2011.

24. Vinuesa CG, Rigby RJ and Yu D: Logic and extent of miRNA-mediated control of autoimmune gene expression. Int Rev Immunol 28: 112-138, 2009.

25. Benson AB III, Venook AP, Bekaii-Saab T, Chan E, Chen YJ, Cooper HS, Engstrom PF, Enzinger PC, Fenton MJ, Fuchs CS, et al; National Comprehensive Cancer Network: Colon cancer, version 3.2014. J Natl Compr Canc Netw 12: 1028-1059, 2014.

26. Livak KJ and Schmittgen TD: Analysis of relative gene expression data using real-time quantitative PCR and the 2(-Delta Delta C(T)) method. Methods 25: 402-408, 2001.

27. Belyavsky A, Vinogradova T and Rajewsky K: PCR-based cDNA library construction: General cDNA libraries at the level of a few cells. Nucleic Acids Res 17: 2919-2932, 1989.

28. Esquela-Kerscher A and Slack FJ: Oncomirs-microRNAs with a role in cancer. Nat Rev Cancer 6: 259-269, 2006.

29. Bueno MJ and Malumbres M: MicroRNAs and the cell cycle. Biochim Biophys Acta 1812: 592-601, 2011.

30. Lieberman J, Slack F, Pandolfi PP, Chinnaiyan A, Agami R and Mendell JT: Noncoding RNAs and cancer. Cell 153: 9-10, 2013.

31. Kai-ming $\mathrm{Wu}, \mathrm{Yu}-$ long He, Fa-keng Liu and Jian-hui Chen: Expression of mitochondrial transcription factor a in colon cancer and its role for proliferative regulation. Chinese Journal Of Bases And Clinics In General Surgery 22: 576-580, 2015. 